\title{
Risk factors for malnutrition among school-aged children: a cross-sectional study in rural Madagascar
}

\author{
Hirotsugu Aiga ${ }^{1,2^{*}}$ (D, Kanae Abe ${ }^{3}$, Vonjy Nirina Andrianome ${ }^{4}$, Emmanuel Randriamampionona ${ }^{5}$,
} Angèle Razafitompo Razafinombana ${ }^{3}$, Toshiyasu Murai ${ }^{6}$ and Masahiro Hara ${ }^{3}$

\begin{abstract}
Background: For over 20 years, Madagascar has been challenged by continued high prevalence of stunting, underweight and wasting among children under 5 years of age. Yet, nutritional status of post-under-five age group has never been assessed in the country, despite its importance in relation not only to physical health but also to cognitive capacity and educational achievements of children. This study aims to estimate prevalence of malnutrition among schoolchildren aged 5-14 years in Madagascar. It further attempts to identify the possible risk factors for their malnutrition. This is the first study that estimates prevalence of malnutrition among school-aged children in Madagascar.
\end{abstract}

Methods: A cross-sectional household survey was conducted in Antananarivo-Avaradrano district, Analamanga region, Madagascar. The study targeted 393 first and second graders 5-14 years of age enrolled at 10 primary schools, where school-feeding was implemented. Data were collected from anthropometric measurements, their subsequent household structured interviews and observations. Bivariate (Chi-square test or Mann-Whitney's U test) and multivariable (logistic regression) analyses were performed, to identify the possible risk factors associated with malnutrition.

Results: The overall prevalence rates of stunting, underweight and thinness were 34.9\%, 36.9\% and $11.2 \%$, respectively. Nineteen children (4.8\%) suffered from all the three forms of undernutrition. Older schoolchildren had a significantly greater likelihood of being stunted, underweight and thin. The greater number of members a household had, the higher likelihood of being stunted and thin its schoolchild had. Children having lower Household Dietary Diversity Score were more likely to be underweight. Yet, 'Had lunch at school yesterday was associated neither with being stunted nor with being underweight and thin. This implies room for improvement of the current school feeding program.

Conclusions: Prevalence rates of stunting and underweight among 393 children examined were as high as the national averages among children under 5 years of age. Adequate food availability and dietary diversity over a sufficient period (incl. 5-14 years of age) are necessary for increasing likelihood of catch-up in height-for-age and weight-for-age, which are expectable during adolescence. To supplement inadequate household dietary diversity practices, school-feeding program may need to use more animal-protein ingredients.

Keywords: Madagascar, Malnutrition, School-aged children, School feeding

\footnotetext{
*Correspondence: aiga.hirotsugu@jica.go.jp; hiroaiga@gwu.edu

'Human Development Department, Japan International Cooperation Agency

(JICA), 3rd floor, Nibancho Center Building, 5-25 Niban-cho, Chiyoda-ku,

Tokyo 102-8012, Japan

2Department of Global Health, Milken Institute School of Public Health, The

George Washington University, 950 New Hampshire Ave, NW, 7th floor,

Washington, DC 20052, USA

Full list of author information is available at the end of the article
}

(c) The Author(s). 2019 Open Access This article is distributed under the terms of the Creative Commons Attribution 4.0 International License (http://creativecommons.org/licenses/by/4.0/), which permits unrestricted use, distribution, and reproduction in any medium, provided you give appropriate credit to the original author(s) and the source, provide a link to the Creative Commons license, and indicate if changes were made. The Creative Commons Public Domain Dedication waiver (http://creativecommons.org/publicdomain/zero/1.0/) applies to the data made available in this article, unless otherwise stated. 


\section{Background}

Nutrition continues to be a key global development agenda under the Sustainable Development Goals (SDGs) after the completion of the Millennium Development Goals (MDGs) era. Despite a critical need for addressing malnutrition among all populations, the monitoring indicators currently in SDG2 and previously in MDG1 are consistently focused on children under 5 years of age $[1,2]$. While admitting children under 5 years of age serve not only as the highest priority subpopulation but also as a leading proxy for entire populations in monitoring nations' nutritional status, it is necessary to capture broader age groups. Scaling Up Nutrition (SUN), a global multisectoral movement on nutrition, addresses malnutrition in a more comprehensive manner, by expanding its target monitoring groups beyond children under 5 years of age (children 13-15 years of age, women 15-49 years of age, and adults $\geq 18$ years of age) [3]. Yet, schoolchildren aged 5-14 years remain excluded from the monitoring frameworks of these major global development agendas as if they were a neglected sub-population. As a result, school-aged children are rarely targeted in nutrition surveys, despite significant impacts of nutritional status on their health, cognition, educational achievements and future economic productivity $[4,5]$.

Malnutrition among children under 5 years of age has been regularly monitored in all countries since the WHO's launch of the standard growth chart in 1978 [6]. Yet, it was just 2007 when WHO launched the standard growth chart for children and adolescents aged 5-19 years, by applying prevalence of stunting, underweight, and not wasting but thinness as the malnutrition indicators [7]. The long-term absence of the technical tools for monitoring the three malnutrition dimensions until 2007 might have discouraged researchers from conducting studies on malnutrition among school-aged children. Recently, World Bank started highlighting a need for addressing malnutrition among school-aged children as a foundational investment a country should make, by launching the Human Capital Measurement Project [5]. Thus, a global momentum is being created toward more studies on malnutrition among school-aged children.

For over 20 years, Madagascar has been challenged by consistently higher prevalence of stunting among children under 5 years of age (around 50\%) for decades. The latest data $(49.2 \%$ as of 2016$)$ is the fifth highest in the world [8]. To address the country's long-lasting high malnutrition prevalence, the Government of Madagascar increasingly focuses on multi-sectoral coordination for greater coverage and impacts of the national nutrition program, by launching the third National Nutrition Action Plan (PNAN III). PNAN III also calls for multisectoral interventions for school-aged children, e.g. community-based deworming, school feeding, household food security, and crop diversification [9]. As a subcomponent policy of PNAN III, National School Feeding and Nutrition Strategy (SNANS) specifies the school-based nutrition interventions. Nevertheless, even basic malnutrition indicators among school-aged children (i.e. prevalence of stunting, underweight, thinness, overweight and obesity) have been neither analyzed nor reported. It is generally difficult for stunted children to catch up during 24-59 months of age [10]. Therefore, the malnutrition status among children under 5 years of age in Madagascar implies the equally high malnutrition prevalence among those $\geq 5$ years of age, due to chronic food insecurity and inadequate feeding practices in the country [11].

In view of unknown but presumably higher malnutrition among school-aged children, the Malagasy Ministry of National Education (MoNE), in collaboration with Japan International Cooperation Agency (JICA), has been committed to strengthening the community-based school feeding program, by developing and piloting its effective and sustainable model. Basic framework of the program defines community-based school feeding as regular provision of meals on school days using locally either produced, procured or donated food items through a school management committee. This study provides the program with the baseline data which not only inform its detailed design but also will be compared with end-line data in the future.

This study is aimed at estimating malnutrition prevalence among children enrolled in rural public primary schools in Madagascar, where school-feeding is implemented, as the pre-intervention baseline data. It further attempts to identify the possible risk factors for malnutrition among them. This study is expected to contribute to informing the design of the community-based school feeding program, by making evidence-based recommendations on it.

\section{Methods}

A cross-sectional household survey was conducted, to estimate malnutrition prevalence (i.e. stunting, underweight, thinness, overweight and obesity) among schoolaged children in Antananarivo-Avaradrano district, Analamanga region, Madagascar. Note that stunting and underweight among children 5-19 years of age are defined in the same manner as those among children under 5 years of age (i.e. by employing z-scores for height-for-age and weight-for-age). Yet, thinness among children 5-19 years of age is differently defined from wasting among children under 5 years of age, though both are aimed at measuring acute malnutrition in common. Thinness is defined as those having a $\mathrm{z}$-score $<-2$ for BMI-for-age, while wasting is defined as those having 
a z-score $<-2$ for weight-for-height [7]. Moreover, overweight and obesity among children 5-19 years of age are defined as those having $\mathrm{z}$-scores $>1$ and $>2$ respectively for BMI-for-age, while definitions of overweight and obesity among children under 5 years of age are based on z-scores for weight-for-height.

\section{Study area}

Antananarivo-Avaradrano district is a typical mountainous area located in central inland Madagascar, where temperature is relatively stable all year around (minimum $10^{\circ} \mathrm{C}$ - maximum $27^{\circ} \mathrm{C}$ ). Eighty-one percent of population of the district live in rural area. Average household size is 4.9 members per household and the proportion of male-headed households is $80.1 \%$ [12]. Locally produced food crops include rice, cassava, maize, sweet potato, beans and potatoes, accounting for more than $95 \%$ of cultivated areas [13].

\section{Study group}

It was reported to the MoNE that local-food-based school feeding was implemented at approximately 50 public primary schools in Antananarivo-Avaradrano district. Through conducting document reviews and its subsequent systematic school visits, it was confirmed that 10 of them were actually implementing local-foodbased school feeding (yet only on a very limited scale) as of August 2017, i.e. Ambohimarina, Ambohitrarahaba, Ambohitsoa, Ambovona, Ankadinandriana, Isahafa, Ikianja, Soavinandriamanitra, Tsarahasina, and Viliahazo primary schools. Therefore, all 10 primary schools were targeted in the study. The other 40 primary schools were not implementing school feeding, primarily due to relatively poorer schools' commitments. Of all children enrolled in the 10 schools, exclusively first and second graders were targeted in this study, for the three reasons. First, targeting first and second graders would help minimize the number of dropouts in the postintervention survey. This is because they will be more contactable at the time of post-intervention survey to be conducted 4-5 years after strengthening local-foodbased school feeding starts. They are likely to stay enrolled in the same schools without going on to secondary schools or getting a job elsewhere. Second, a majority of first and second graders have just entered post-under-five age group, in which the likelihood of recovering from stunting might be expected $[14,15]$. Third, as AnthroPlus, a WHO's software, allows zscores for weight-for-age to be calculated only for children up to 120.8 months of age [16], first and second graders who are overall younger than that age should be targeted.

\section{Sample size and sampling methods}

Prevalence of malnutrition (i.e. stunting, underweight, thinness, overweight and obesity) among school-aged children was previously reported neither from Antananarivo-Avaradrano district nor from any other parts of Madagascar. National prevalence of stunting among children under 5 years of age has been around $50 \%$ for decades [8]. Therefore, we assumed that the prevalence among first and second graders could be $50 \%$, under which sample size becomes the greatest (i.e. sufficient sample size also for estimating prevalence rates of underweight, thinness, overweight and obesity). To estimate prevalence representative of children 5-14 years of age enrolled in public primary schools implementing local-food-based school feeding in AntananarivoAvaradrano district, the sample size was calculated with $\alpha \quad$ (error) $=0.05$ and $d$ (precision $)=0.05$. Thus, 384 school-aged children were determined as the required sample size. Assuming 5\% of non-response rate [17], 404 children were employed as the final sample size. To ensure equal representativity of children in each stratum (school, grade and sex), probability-proportional-to-size sampling was applied (Table 1) [7, 8, 18-20]. A total of 404 first and second graders were randomly selected from the respective strata, using student registries of the 10 primary schools.

\section{Data collection}

Anthropometric measurements of sampled first and second graders and structured interviews with their parents were conducted during the period from 20 November to 11 December 2017, early rainy season in Madagascar. Weight and height of children were measured in classrooms of their primary schools by enumerators. Weight measurements were undertaken to the nearest $0.1 \mathrm{~kg}$ using the electronic scale for mother/child $150 \mathrm{~kg}$ x $100 \mathrm{~g}$ (UNICEF reference model: S0141021). Height was measured to the nearest $0.1 \mathrm{~cm}$, using the UNICEF standard height scale. To precisely calculate age of children, their dates of birth were collected from student registries. Data of food items used for lunch served at schools on the previous day were collected from school feeding program records at each primary school.

Subsequently to data collection at schools, children's households were visited to collect data of background variables for malnutrition. A total of 21 background variables were employed to examine their associations with malnutrition among school-aged children (Tables 2 and 3). These background variables were selected primarily from those representing three categories of underlying causes of childhood undernutrition (i.e. household food insecurity, inadequate caring and feeding practices, and unhealthy living environment) [21-23]. The questions on those background variables were included in the structured 
Table 1 Prevalence of malnutrition among schoolchildren 5-14 years of age

\begin{tabular}{|c|c|c|c|c|c|c|c|c|c|c|c|}
\hline \multirow[t]{2}{*}{ Primary school } & \multirow[t]{2}{*}{$N$} & \multicolumn{2}{|c|}{$\begin{array}{l}\text { Stunting } \\
n=393\end{array}$} & \multicolumn{2}{|c|}{$\begin{array}{l}\text { Underweight }{ }^{\mathrm{b}} \\
n=382\end{array}$} & \multicolumn{2}{|c|}{$\begin{array}{l}\text { Thinness } \\
N=393\end{array}$} & \multicolumn{2}{|c|}{$\begin{array}{l}\text { Overweight }^{d} \\
N=393\end{array}$} & \multicolumn{2}{|c|}{$\begin{array}{l}\text { Obsesity } \\
N=393\end{array}$} \\
\hline & & $\bar{n}$ & (\%) & $\bar{n}$ & (\%) & $\bar{n}$ & (\%) & $\bar{n}$ & (\%) & $n$ & (\%) \\
\hline Ambohimarina & 25 & 9 & 36.0 & 7 & 30.4 & 2 & 8.0 & 1 & 4.0 & 0 & 0.0 \\
\hline Ambohitrarahaba & 106 & 33 & 31.1 & 36 & 34.6 & 10 & 9.4 & 1 & 0.9 & 0 & 0.0 \\
\hline Ambohitsoa & 38 & 11 & 28.9 & 10 & 27.8 & 3 & 7.9 & 0 & 0.0 & 0 & 0.0 \\
\hline Ambovona & 9 & 5 & 55.6 & 5 & 55.6 & 3 & 33.3 & 1 & 11.1 & 0 & 0.0 \\
\hline Ankadinandriana & 21 & 10 & 47.6 & 9 & 42.9 & 1 & 4.5 & 0 & 0.0 & 0 & 0.0 \\
\hline Isahafa & 45 & 12 & 26.7 & 14 & 31.8 & 2 & 4.4 & 1 & 2.2 & 0 & 0.0 \\
\hline Ikianja & 31 & 13 & 41.9 & 12 & 41.4 & 5 & 16.1 & 0 & 0.0 & 0 & 0.0 \\
\hline Soavinandriamanitra & 35 & 14 & 40.0 & 15 & 45.5 & 5 & 14.3 & 0 & 0.0 & 0 & 0.0 \\
\hline Tsarahasina & 50 & 18 & 36.0 & 20 & 40.0 & 5 & 10.0 & 0 & 0.0 & 0 & 0.0 \\
\hline Viliahazo & 33 & 12 & 36.4 & 17 & 51.5 & 8 & 24.2 & 0 & 0.0 & 0 & 0.0 \\
\hline \multirow[t]{2}{*}{ Total } & 393 & 137 & 34.9 & 145 & 36.9 & 44 & 11.2 & 4 & 1.0 & 0 & 0.0 \\
\hline & & \multicolumn{2}{|c|}{ 95\% Cl: 30.4-39.7 } & \multicolumn{2}{|c|}{$95 \%$ Cl: 32.2-41.9 } & \multicolumn{2}{|c|}{ 95\% Cl: 8.5-14.7 } & \multicolumn{2}{|c|}{$95 \%$ Cl: 0.4-2.6 } & \multicolumn{2}{|c|}{$95 \%$ Cl: $0.0-1.0$} \\
\hline \multicolumn{2}{|c|}{ National prevalence among children under 5 years of age } & \multicolumn{2}{|l|}{$49.2^{f}$} & \multicolumn{2}{|c|}{$36.0^{9}$} & \multicolumn{2}{|c|}{$(\text { n.a. })^{\mathrm{h}}$} & \multicolumn{2}{|l|}{$6.2^{i}$} & \multicolumn{2}{|c|}{$(\text { n.a. })^{h}$} \\
\hline
\end{tabular}

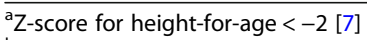

bZ-score for weight-for-age <-2 [7]. As WHO's growth reference does not cover children 121 months of age and older, 11 children categorized into the age group were excluded from estimation of prevalence of underweight [18]

'Z-score for BMI-for-age $<-2$ [7]

${ }^{\mathrm{d}} 2>\mathrm{Z}$-score for BMl-for-age $>1$ [7]

eZ-score for BMl-for-age $>2$ [7]

${ }^{\mathrm{f}}$ Nationally representative prevalence among children under 5 years of age as of 2015 (IFPRI, 2016) [8]

${ }^{9}$ Nationally representative prevalence among children under 5 years of age as of 2008-2012 (UNICEF, 2018) [19]

${ }^{\mathrm{h}}$ Thinness and obesity are not internationally employed as malnutrition indicators for children under 5 years of age

'Nationally representative prevalence among children under 5 years of age as of 2004 (World Bank, 2018) [20]

questionnaire (see Additional file 1). Of them, the data on types of drinking water source, types of toilet, presence of soap/ash for handwashing, food storage, and utensil maintenance were collected through enumerators' observations. The data on other variables were collected through interviews with parents and other household members responsible for food preparation (incl. Children's food consumption data based on 24-h dietary recall). Household Dietary Diversity Score (HDDS) was further calculated by summing up the number of food groups consumed by the children during last $24 \mathrm{~h}$ [21]. Dietary diversity is a qualitative measure of food consumption that reflects household's access to a variety of foods, and serves as a proxy for both macro- and micro-nutrient adequacy of the diet of individuals [21]. School feeding can not only complement inadequate household dietary diversity by providing children with food items not being often consumed at their households, but also trigger households' dietary behavior changes [24].

Interviews were undertaken by asking the questions in Malagasy, the local language of Madagascar. Two repeated household visits were made, when parents and other household members were absent. The questionnaire was completed by the enumerator during an interview, and later field-checked. Eighteen locally recruited skilled enumerators were trained on anthropometric measurement, interviewing and observation, and supervised by four field team leaders.

\section{Data analysis}

The data obtained through household interviews, observations and anthropometric measurements were entered into a microcomputer. By using AnthroPlus (WHO, Geneva) [16] and $R$ for Mac OS $X$ version 3.2.2 (R Foundation for Statistical Computing, Vienna) [25], z-scores for height-for-age, weight-for-age and BMI-for-age were calculated based on the WHO standard reference population 5-19 years of age [7, 18]. In Madagascar, children under 5 years of age are sometimes enrolled in first or second graders due to the country's flexible and less enforced age requirements for primary education enrollment. Those aged under 5 years of age were excluded from the analysis, since they are out of the scope of this study. The statistical analyses other than z-score calculations were conducted, by using SPSS for Windows, version 22 (IBM/SPSS Inc., Chicago) [26].

Both bivariate and multivariable analyses were undertaken to identify the background variables (independent variables) associated with whether being malnourished (dependent variable). While the dependent variables are dichotomous, the independent variables are composed of interval/ratio variables and categorical variables. 
Table 2 Bivariate analyses between schoolchildren's malnutrition and its categorical background variables

\begin{tabular}{|c|c|c|c|c|c|c|c|c|c|c|c|c|c|c|c|}
\hline \multirow[t]{3}{*}{ Background variable } & \multicolumn{5}{|c|}{ Stunting $(N=393)$} & \multicolumn{5}{|c|}{ Underweight $(N=382)$} & \multicolumn{5}{|c|}{ Thinness $(N=393)$} \\
\hline & \multicolumn{2}{|c|}{ (+) Stunted } & \multicolumn{2}{|c|}{$\begin{array}{l}\text { (-) Not } \\
\text { stunted }\end{array}$} & \multirow[t]{2}{*}{$\overline{P \text {-value }}{ }^{a}$} & \multicolumn{2}{|c|}{$\begin{array}{l}\text { (+) Under- } \\
\text { weight }\end{array}$} & \multicolumn{2}{|c|}{$\begin{array}{l}\text { (-) Not } \\
\text { underweight }\end{array}$} & \multirow[t]{2}{*}{$P$-value ${ }^{a}$} & \multicolumn{2}{|c|}{ (+) Thin } & \multicolumn{2}{|c|}{$\begin{array}{l}\text { (-) Not } \\
\text { thin }\end{array}$} & \multirow[t]{2}{*}{$P$-value } \\
\hline & $n$ & (\%) & $N$ & $(\%)$ & & $n$ & $(\%)$ & $n$ & $(\%)$ & & $n$ & $(\%)$ & $n$ & $(\%)$ & \\
\hline \multicolumn{16}{|l|}{$v_{1}:$ Sex } \\
\hline Male & 75 & 54.7 & 135 & 52.7 & 0.751 & 83 & 57.2 & 121 & 51.1 & 0.247 & 25 & 56.8 & 185 & 53.0 & 0.749 \\
\hline Female & 62 & 45.3 & 121 & 47.3 & & 62 & 42.8 & 116 & 48.9 & & 19 & 43.2 & 164 & 47.0 & \\
\hline Total & 137 & 100 & 256 & 100 & & 145 & 100 & 237 & 100 & & 44 & 100 & 349 & 100 & \\
\hline \multicolumn{16}{|l|}{$\mathrm{v}_{2}$ : Diarrhea during last 14 days } \\
\hline With diarrhea & 4 & 2.9 & 8 & 3.1 & 1.000 & 5 & 3.4 & 7 & 3.0 & 0.771 & 2 & 4.5 & 10 & 2.9 & 0.632 \\
\hline Without diarrhea & 133 & 97.1 & 252 & 96.9 & & 140 & 96.6 & 230 & 97.0 & & 42 & 95.5 & 339 & 97.1 & \\
\hline Total & 137 & 100 & 256 & 100 & & 145 & 100 & 237 & 100 & & 44 & 100 & 349 & 100 & \\
\hline \multicolumn{16}{|l|}{$v_{3}:$ Took deworming tables at school } \\
\hline Took & 53 & 38.7 & 99 & 38.7 & 1.000 & 63 & 43.2 & 85 & 35.9 & 0.160 & 16 & 36.4 & 136 & 39.0 & 0.870 \\
\hline Did not take & 84 & 61.3 & 157 & 61.3 & & 82 & 56.8 & 152 & 64.1 & & 28 & 63.6 & 213 & 61.0 & \\
\hline Total & 137 & 100 & 256 & 100 & & 145 & 100 & 237 & 100 & & 44 & 100 & 349 & 100 & \\
\hline \multicolumn{16}{|l|}{ Major income sources } \\
\hline Daily job & 46 & 33.6 & 93 & 36.7 & Ref. & 44 & 30.3 & 90 & 37.9 & Ref. & 16 & 36.4 & 122 & 35.7 & Ref. \\
\hline$v_{4}$ : Agriculture or crop sales & 35 & 25.5 & 83 & 32.4 & 0.137 & 42 & 29.0 & 73 & 30.8 & 0.731 & 10 & 22.7 & 110 & 30.9 & 0.299 \\
\hline$v_{5}:$ Seller, trader or commercial business & 23 & 16.8 & 22 & 8.6 & $0.020^{*}$ & 26 & 17.9 & 18 & 7.6 & $0.003^{* *}$ & 7 & 15.9 & 38 & 10.9 & 0.317 \\
\hline$v_{6}$ : Skilled wage labor & 9 & 6.6 & 19 & 7.4 & 0.839 & 9 & 6.2 & 19 & 8.0 & 0.551 & 0 & 0.0 & 28 & 8.0 & 0.058 \\
\hline$\vee_{7}$ : Unskilled wage labor & 12 & 8.8 & 14 & 5.5 & 0.286 & 11 & 7.6 & 15 & 6.3 & 0.687 & 5 & 11.4 & 21 & 6.0 & 0.193 \\
\hline$v_{8}:$ Small-scale industry or handicraft & 8 & 5.8 & 18 & 6.6 & 0.831 & 8 & 5.5 & 16 & 6.8 & 0.671 & 5 & 11.4 & 20 & 5.7 & 0.180 \\
\hline$v_{9}$ : Livestock or animal sales & 1 & 0.7 & 4 & 1.6 & 0.662 & 2 & 1.4 & 3 & 1.3 & 1.000 & 0 & 0.0 & 5 & 1.4 & 1.000 \\
\hline$v_{10}$ : Others & 3 & 2.2 & 3 & 1.2 & 0.424 & 3 & 2.1 & 3 & 1.3 & 0.678 & 1 & 2.3 & 5 & 1.4 & 0.512 \\
\hline Total & 137 & 100 & 256 & 100 & & 145 & 100 & 237 & 100 & & 44 & 100 & 349 & 100 & \\
\hline \multicolumn{16}{|l|}{$\vee_{11}$ : Land ownership } \\
\hline Own land for housing or farming & 101 & 73.7 & 207 & 80.9 & 0.123 & 111 & 76.6 & 188 & 79.3 & 0.526 & 33 & 75.0 & 275 & 78.8 & 0.562 \\
\hline Don't own land for housing or farming & 36 & 26.3 & 49 & 19.1 & & 34 & 23.4 & 49 & 20.7 & & 11 & 25.0 & 75 & 21.2 & \\
\hline Total & 137 & 100 & 256 & 100 & & 145 & 100 & 237 & 100 & & 44 & 100 & 349 & 100 & \\
\hline \multicolumn{16}{|c|}{ Education attainment of household member responsible for food preparation } \\
\hline$v_{12}:$ Never gone to school & 6 & 4.4 & 9 & 3.5 & 0.783 & 6 & 4.1 & 9 & 3.8 & 1.000 & 0 & 0.0 & 15 & 4.3 & 0.392 \\
\hline Dropped out from primary school & 72 & 52.5 & 112 & 44.1 & Ref. & 70 & 48.3 & 107 & 45.2 & Ref. & 22 & 50.0 & 163 & 46.7 & Ref. \\
\hline $\mathrm{v}_{13}:$ Primary school & 49 & 35.8 & 100 & 39.1 & 0.586 & 59 & 40.7 & 88 & 37.1 & 0.516 & 16 & 36.4 & 133 & 38.1 & 0.870 \\
\hline$v_{14}:$ Secondary school & 8 & 5.8 & 29 & 11.3 & 0.102 & 10 & 6.9 & 26 & 11.0 & 0.210 & 6 & 13.6 & 31 & 8.9 & 0.282 \\
\hline$v_{15}:$ High school & 2 & 1.5 & 4 & 1.6 & 1.000 & 0 & 0.0 & 6 & 2.5 & 0.087 & 0 & 0.0 & 6 & 1.7 & 1.000 \\
\hline$v_{16}$ : University or other higher education & 0 & 0.0 & 2 & 0.4 & 1.000 & 0 & 0.0 & 1 & 0.4 & 1.000 & 0 & 0.0 & 1 & 0.3 & 1.000 \\
\hline Total & 137 & 100 & 256 & 100 & & 145 & 100 & 237 & 100 & & 44 & 100 & 349 & 100 & \\
\hline$v_{17}:$ Type of source of water & & & & & & & & & & & & & & & \\
\hline Improved type of source of water ${ }^{b}$ & 61 & 44.5 & 109 & 42.6 & 0.749 & 57 & 39.3 & 107 & 45.1 & 0.288 & 19 & 43.2 & 151 & 43.3 & 1.000 \\
\hline Not improved type of source of water ${ }^{c}$ & 76 & 55.5 & 147 & 57.4 & & 88 & 60.7 & 130 & 54.9 & & 25 & 56.8 & 198 & 56.7 & \\
\hline Total & 137 & 100 & 256 & 100 & & 145 & 100 & 237 & 100 & & 44 & 100 & 349 & 100 & \\
\hline$v_{18}:$ Type of sanitation facility & & & & & & & & & & & & & & & \\
\hline Improved type of sanitation facility ${ }^{d}$ & 7 & 5.1 & 16 & 6.3 & 0.622 & 7 & 4.8 & 16 & 6.8 & 0.512 & 3 & 6.8 & 20 & 5.7 & 0.733 \\
\hline Not improved type of sanitation facility e & 132 & 94.9 & 240 & 93.8 & & 138 & 95.2 & 221 & 93.2 & & 41 & 93.2 & 329 & 94.3 & \\
\hline Total & 137 & 100 & 256 & 100 & & 145 & 100 & 237 & 100 & & 44 & 100 & 349 & 100 & \\
\hline
\end{tabular}


Table 2 Bivariate analyses between schoolchildren's malnutrition and its categorical background variables (Continued)

\begin{tabular}{|c|c|c|c|c|c|c|c|c|c|c|c|c|c|c|c|}
\hline \multirow[t]{3}{*}{ Background variable } & \multicolumn{5}{|c|}{ Stunting $(N=393)$} & \multicolumn{5}{|c|}{ Underweight $(N=382)$} & \multicolumn{5}{|c|}{ Thinness $(N=393)$} \\
\hline & \multicolumn{2}{|c|}{ (+) Stunted } & \multicolumn{2}{|c|}{$\begin{array}{l}\text { (-) Not } \\
\text { stunted }\end{array}$} & \multirow[t]{2}{*}{$P$-value ${ }^{a}$} & \multicolumn{2}{|c|}{$\begin{array}{l}\text { (+) Under- } \\
\text { weight }\end{array}$} & \multicolumn{2}{|c|}{$\begin{array}{l}\text { (-) Not } \\
\text { underweight }\end{array}$} & \multirow[t]{2}{*}{$P$-value ${ }^{a}$} & \multicolumn{2}{|c|}{ (+) Thin } & \multicolumn{2}{|c|}{$\begin{array}{l}(-) \text { Not } \\
\text { thin }\end{array}$} & \multirow[t]{2}{*}{$P$-value ${ }^{a}$} \\
\hline & $n$ & (\%) & N & (\%) & & $n$ & (\%) & $n$ & (\%) & & $n$ & $(\%)$ & $n$ & (\%) & \\
\hline \multicolumn{16}{|c|}{$v_{19}:$ Hand-washing after toilet of household member responsible for food preparation } \\
\hline Wash hands after toilet & 36 & 26.3 & 76 & 29.7 & 0.558 & 40 & 27.6 & 71 & 30.0 & 0.644 & 17 & 38.6 & 95 & 27.2 & 0.155 \\
\hline Don't wash hands after toilet & 101 & 73.7 & 180 & 70.3 & & 105 & 72.4 & 166 & 70.0 & & 27 & 61.4 & 254 & 72.8 & \\
\hline Total & 137 & 100 & 256 & 100 & & 145 & 100 & 237 & 100 & & 44 & 100 & 349 & 100 & \\
\hline \multicolumn{16}{|c|}{$v_{20}$ : Hand-washing before cooking of household member responsible for food preparation } \\
\hline Wash hands before cooking & 33 & 24.1 & 66 & 25.8 & 0.807 & 37 & 25.5 & 61 & 25.7 & 1.000 & 14 & 31.8 & 85 & 24.4 & 0.275 \\
\hline Don't wash hands before cooking & 104 & 75.9 & 190 & 74.2 & & 108 & 74.5 & 176 & 74.3 & & 30 & 68.2 & 264 & 75.6 & \\
\hline Total & 137 & 100 & 256 & 100 & & 145 & 100 & 237 & 100 & & 44 & 100 & 349 & 100 & \\
\hline \multicolumn{16}{|l|}{$v_{21}$ : Hand-washing before eating } \\
\hline Wash hands before cooking & 33 & 24.1 & 70 & 27.3 & 0.548 & 36 & 24.8 & 66 & 27.8 & 0.553 & 14 & 31.8 & 89 & 25.5 & 0.367 \\
\hline Don't wash hands before cooking & 104 & 75.9 & 186 & 72.7 & & 109 & 75.2 & 171 & 72.2 & & 30 & 68.2 & 260 & 74.5 & \\
\hline Total & 137 & 100 & 256 & 100 & & 145 & 100 & 237 & 100 & & 44 & 100 & 349 & 100 & \\
\hline \multicolumn{16}{|l|}{ Rice storage } \\
\hline Plastic/metal container with cover & 40 & 29.2 & 106 & 41.3 & Ref. & 40 & 27.5 & 104 & 43.9 & Ref. & 13 & 29.5 & 133 & 38.1 & Ref. \\
\hline $\mathrm{v}_{22}:$ Plastic/metal container without cover & 1 & 0.7 & 8 & 3.1 & 0.171 & 3 & 2.1 & 5 & 2.1 & 1.000 & 1 & 2.3 & 8 & 2.3 & 1.000 \\
\hline$\vee_{23}: 50 \mathrm{~kg}$ rice bag & 32 & 23.4 & 45 & 17.6 & 0.183 & 28 & 19.3 & 46 & 19.4 & 1.000 & 9 & 20.5 & 68 & 19.5 & 0.842 \\
\hline$\vee_{24}:$ Handbasket bag & 35 & 25.5 & 48 & 18.8 & 0.121 & 38 & 26.2 & 42 & 17.7 & 0.053 & 13 & 29.5 & 70 & 20.1 & 0.169 \\
\hline $\mathrm{V}_{25}:$ Plastic bag & 19 & 13.9 & 24 & 9.4 & 0.179 & 23 & 15.9 & 20 & 8.4 & $0.030^{*}$ & 5 & 11.4 & 38 & 10.9 & 1.000 \\
\hline$v_{26}$ : None (buy rice only for the day) & 9 & 6.6 & 22 & 8.6 & 0.599 & 12 & 8.3 & 17 & 7.2 & 0.695 & 3 & 6.8 & 28 & 8.0 & 1.000 \\
\hline$v_{27}:$ Others & 1 & 0.7 & 3 & 1.2 & 1.000 & 1 & 0.7 & 3 & 1.3 & 1.000 & 0 & 0.0 & 4 & 1.1 & 1.000 \\
\hline Total & 137 & 100 & 256 & 100 & & 145 & 100 & 237 & 100 & & 44 & 100 & 349 & 100 & \\
\hline \multicolumn{16}{|l|}{ Utensil maintenance } \\
\hline$v_{28}:$ In cabinet after cleaning & 23 & 16.8 & 45 & 17.6 & 0.889 & 23 & 15.9 & 44 & 18.6 & 0.580 & 7 & 15.9 & 61 & 17.5 & 1.000 \\
\hline In open-space after cleaning & 69 & 50.4 & 123 & 48.0 & Ref. & 74 & 51.0 & 111 & 46.8 & Ref. & 25 & 56.8 & 167 & 47.9 & Ref. \\
\hline $\mathrm{v}_{29}$ : Covered with cloth after cleaning & 28 & 20.4 & 47 & 18.4 & 0.686 & 29 & 20.0 & 43 & 18.1 & 0.684 & 10 & 22.7 & 65 & 18.6 & 0.542 \\
\hline$v_{30}:$ In open-space without cleaning & 17 & 12.4 & 41 & 16.0 & 0.373 & 19 & 13.1 & 39 & 16.5 & 0.463 & 2 & 4.5 & 56 & 16.0 & $0.042^{*}$ \\
\hline Total & 137 & 100 & 256 & 100 & & 145 & 100 & 237 & 100 & & 44 & 100 & 349 & 100 & \\
\hline \multicolumn{16}{|l|}{ Meals taken yesterday } \\
\hline Breakfast, lunch and dinner/supper & 135 & 98.6 & 254 & 99.2 & Ref. & 144 & 99.3 & 234 & 98.8 & Ref. & 44 & 100 & 345 & 98.8 & Ref. \\
\hline$v_{31}$ : Breakfast and lunch & 1 & 0.7 & 0 & 0.0 & 0.349 & 1 & 0.7 & 0 & 0.0 & 0.380 & 0 & 0.0 & 1 & 0.3 & 1.000 \\
\hline$v_{32}:$ Lunch and dinner/supper & 1 & 0.7 & 0 & 0.0 & 0.349 & 0 & 0.0 & 1 & 0.4 & 1.000 & 0 & 0.0 & 1 & 0.3 & 1.000 \\
\hline$v_{33}:$ Only breakfast & 0 & 0.0 & 2 & 0.8 & 0.545 & 0 & 0.0 & 2 & 0.8 & 0.528 & 0 & 0.0 & 2 & 0.6 & 1.000 \\
\hline Total & 137 & 100 & 256 & 100 & & 145 & 100 & 237 & 100 & & 44 & 100 & 349 & 100 & \\
\hline $\mathrm{V}_{34}:$ Yesterday's lunch at school & & & & & & & & & & & & & & & \\
\hline $\begin{array}{l}\text { Had lunch through school feeding } \\
\text { program }\end{array}$ & 25 & 18.2 & 47 & 18.4 & 1.000 & 24 & 16.6 & 47 & 19.8 & 0.498 & 7 & 15.9 & 65 & 18.6 & 0.836 \\
\hline Did not have lunch at school & 113 & 81.8 & 209 & 81.6 & & 121 & 83.4 & 190 & 80.2 & & 37 & 84.1 & 284 & 81.4 & \\
\hline Total & 137 & 100 & 256 & 100 & & 145 & 100 & 237 & 100 & & 44 & 100 & 349 & 100 & \\
\hline Food consumption during last $24 \mathrm{~h}^{\mathrm{f}}$ & & & & & & & & & & & & & & & \\
\hline$v_{35}:$ Cereal & 137 & 100 & 256 & 100 & (n.a.) & 145 & 100 & 237 & 100 & (n.a.) & 44 & 100 & 349 & 100 & (n.a.) \\
\hline$v_{36}:$ White tubers and roots & 57 & 41.6 & 97 & 37.9 & 0.516 & 59 & 40.7 & 92 & 38.8 & 0.747 & 135 & 38.7 & 19 & 43.2 & 0.624 \\
\hline$v_{37}:$ Vegetables & 130 & 94.9 & 246 & 96.1 & 0.608 & 136 & 93.8 & 229 & 96.6 & 0.209 & 41 & 93.2 & 335 & 96.0 & 0.421 \\
\hline
\end{tabular}


Table 2 Bivariate analyses between schoolchildren's malnutrition and its categorical background variables (Continued)

\begin{tabular}{|c|c|c|c|c|c|c|c|c|c|c|c|c|c|c|c|}
\hline \multirow[t]{3}{*}{ Background variable } & \multicolumn{5}{|c|}{ Stunting $(N=393)$} & \multicolumn{5}{|c|}{ Underweight $(N=382)$} & \multicolumn{5}{|c|}{ Thinness $(N=393)$} \\
\hline & \multicolumn{2}{|c|}{ (+) Stunted } & \multicolumn{2}{|c|}{$\begin{array}{l}(-) \text { Not } \\
\text { stunted }\end{array}$} & \multirow[t]{2}{*}{$P$-value ${ }^{a}$} & \multicolumn{2}{|c|}{$\begin{array}{l}\text { (+) Under- } \\
\text { weight }\end{array}$} & \multicolumn{2}{|c|}{$\begin{array}{l}(-) \text { Not } \\
\text { underweight }\end{array}$} & \multirow[t]{2}{*}{$P$-value ${ }^{a}$} & \multicolumn{2}{|c|}{ (+) Thin } & \multicolumn{2}{|c|}{$\begin{array}{l}(-) \text { Not } \\
\text { thin }\end{array}$} & \multirow[t]{2}{*}{$P$-value } \\
\hline & $n$ & (\%) & $\bar{N}$ & $\overline{(\%)}$ & & $\bar{n}$ & (\%) & $n$ & (\%) & & $n$ & $(\%)$ & $\bar{n}$ & $(\%)$ & \\
\hline$v_{38}$ : Fruits & 13 & 9.5 & 22 & 8.6 & 0.853 & 14 & 9.7 & 20 & 8.4 & 0.713 & 4 & 9.1 & 31 & 3.9 & 1.000 \\
\hline$v_{39}:$ Meats & 38 & 27.7 & 82 & 32.0 & 0.422 & 33 & 22.8 & 82 & 34.6 & $0.016^{*}$ & 106 & 30.4 & 14 & 31.8 & 0.863 \\
\hline$v_{40}$ : Eggs & 10 & 7.3 & 34 & 13.3 & 0.093 & 32 & 13.5 & 11 & 7.6 & 0.095 & 41 & 11.7 & 3 & 6.8 & 0.450 \\
\hline $\mathrm{v}_{41}$ : Fish and other seafood & 27 & 19.7 & 54 & 21.1 & 0.795 & 26 & 17.9 & 54 & 22.8 & 0.300 & 6 & 13.6 & 75 & 21.5 & 0.322 \\
\hline$v_{42}:$ Legumes, nuts and seeds & 59 & 43.1 & 109 & 42.6 & 1.000 & 63 & 43.4 & 100 & 42.2 & 0.832 & 20 & 45.5 & 148 & 42.4 & 0.748 \\
\hline $\mathrm{V}_{43}:$ Milk and milk products & 10 & 7.3 & 37 & 14.5 & $0.041^{*}$ & 11 & 7.6 & 36 & 15.2 & $0.036^{*}$ & 5 & 11.4 & 42 & 12.0 & 1.000 \\
\hline$v_{44}:$ Oils and fats & 134 & 97.8 & 247 & 96.5 & 0.554 & 141 & 97.2 & 229 & 96.6 & 1.000 & 43 & 97.7 & 338 & 96.8 & 1.000 \\
\hline$v_{45}$ : Sweets & 83 & 60.6 & 184 & 71.9 & $0.024^{*}$ & 93 & 64.1 & 168 & 70.9 & 0.176 & 28 & 63.6 & 239 & 68.5 & 0.499 \\
\hline $\mathrm{V}_{46}$ : Spices, condiments and beverage & 135 & 98.5 & 256 & 100 & 0.121 & 143 & 98.6 & 237 & 100 & 0.143 & 44 & 100 & 347 & 99.4 & 1.000 \\
\hline
\end{tabular}

${ }^{*} P<0.05$

** $P<0.01$

${ }^{\mathrm{a}}$ Chi-square test

${ }^{\mathrm{b}}$ Improved types of source of water include: (i) piped household water connection; (ii) public standpipe; (iii) protected well/borehole; (iv) Protected spring; and (v) rainwater collection

'Not improved types of source of water include: (i) unprotected well; (ii) unprotected spring; (iii) surface water (river, lake, reservoir, dam, canal, irrigation channel); (iv) vendor-provided water (cart, tanker truck); and (v) bottled water

IImproved types of toilet include: (i) flush connected to sewerage system (ii) flush connected to a septic tank; (iii) toilet connected to a pit; (iv) improved ventilated latrine; and (v) latrines with slab

${ }^{e}$ Not improved types of toilet include: (i) flush toilet not connected to sewerage/pit; (ii) latrines without slab/open pit; (iii) bucket; (vi) joint installation with other households (public toilet); and (viii) Outdoor defecation (field, forest, bush and river)

${ }^{f}$ Twelve categories of foods are based on food groups for calculating Household Dietary Diversity Score (HDDS) of Food and Agriculture Organization of the United Nations (FAO) [21]

Therefore, two types of bivariate analyses were employed. First, the associations between 16 categorical variables and whether being malnourished were examined, using Chi-square test. For five categorical variables composed of three or more categories ("Major income sources,' 'Education attainment of household member responsible for food preparation', 'Rice storage', 'Utensil maintenance, and 'Meals taken yesterday'), dummy variables were created. As a result, 46 categorical variables were created (incl. 24 dummy variables). The category with the greatest frequency was designated as the reference for the dummy variables. Second, the associations between five interval/ratio variables and whether being malnourished were examined, using a nonparametric method (Mann-Whitney's U test), as data of those variables were expected not to be normally

Table 3 Bivariate analyses between schoolchildren's malnutrition and its interval/ratio background variables

\begin{tabular}{|c|c|c|c|c|c|c|c|c|c|c|c|c|c|c|c|}
\hline \multirow[t]{3}{*}{ Background variable } & \multicolumn{5}{|c|}{ Stunting $(N=393)$} & \multicolumn{5}{|c|}{ Underweight $(N=382)$} & \multicolumn{5}{|c|}{ Thinness $(N=393)$} \\
\hline & \multicolumn{2}{|c|}{ (+) Stunted } & \multicolumn{2}{|c|}{$\begin{array}{l}(-) \text { Not } \\
\text { stunted }\end{array}$} & \multirow[t]{2}{*}{$P$-value ${ }^{a}$} & \multicolumn{2}{|c|}{$\begin{array}{l}(+) \text { Under- } \\
\text { weight }\end{array}$} & \multicolumn{2}{|c|}{$\begin{array}{l}\text { (-) Not } \\
\text { underweight }\end{array}$} & \multirow[t]{2}{*}{$P$-value ${ }^{a}$} & \multicolumn{2}{|c|}{ (+) Thin } & \multicolumn{2}{|c|}{$\begin{array}{l}(-) \text { Not } \\
\text { thin }\end{array}$} & \multirow[t]{2}{*}{$P$-value } \\
\hline & Mean & s.d. & Mean & s.d. & & Mean & s.d. & Mean & s.d. & & Mean & s.d. & Mean & s.d. & \\
\hline $\mathrm{V}_{47}:$ Age [year] & 7.6 & 1.65 & 7.0 & 1.05 & $<0.001^{* *}$ & 7.4 & 1.12 & 6.8 & 0.99 & $<0.001^{* *}$ & 7.9 & 1.95 & 7.1 & 1.20 & $0.004^{* *}$ \\
\hline $\begin{array}{l}\mathrm{V}_{48} \text { : Total number of household } \\
\text { members [person] }\end{array}$ & 5.9 & 1.82 & 5.2 & 1.65 & $<0.001^{* *}$ & 5.8 & 1.73 & 5.2 & 1.66 & $<0.001^{* *}$ & 6.3 & 2.03 & 5.3 & 1.67 & $0.002^{* *}$ \\
\hline $\begin{array}{l}V_{48} \text { : Access to source of water by } \\
\text { foot [min] }{ }^{\text {b }}\end{array}$ & 3.3 & 3.32 & 3.7 & 3.28 & 0.163 & 3.4 & 3.41 & 3.7 & 3.28 & 0.205 & 3.6 & 2.68 & 3.6 & 3.37 & 0.454 \\
\hline $\begin{array}{l}V_{50} \text { : Access to the nearest health } \\
\text { facility [min] }\end{array}$ & 34.6 & 14.3 & 35.9 & 19.9 & 0.485 & 35.6 & 16.3 & 35.1 & 18.7 & 0.278 & 39.1 & 18.4 & 35.0 & 18.1 & 0.173 \\
\hline $\begin{array}{l}v_{51} \text { : Household dietary diversity } \\
\text { score (HDDS) [pt] }{ }^{c}\end{array}$ & 6.1 & 1.42 & 6.3 & 1.27 & 0.066 & 6.0 & 1.35 & 6.4 & 1.29 & $0.021^{*}$ & 6.2 & 1.49 & 6.3 & 1.31 & 0.851 \\
\hline
\end{tabular}

${ }^{\mathrm{b}}$ The number of minutes spent reaching a water source was measured by allowing an enumerator to walk

'Household Dietary Diversity Score (HDDS) is the score ranging from 0 ( $\mathrm{min})$ to 12 (max), which is calculated by summing up the number of food groups consumed during previous day (24 h) [21] 
distributed. Thus, a total of 51 background variables $(=46$ categorical +5 interval/ratio variables) were employed.

The background variables significantly associated with being malnourished $(P<0.05$ in Chi-square test or Mann-Whitney's U test) were selected as the possible independent variables for multivariable analyses. Prior to applying them to multivariable analyses, multicollinearity between those possible independent variables was examined by assessing variance inflation factor (VIF). Only those with $\mathrm{VIF}<10$ were selected as the independent variables for multivariable analyses. As the dependent variables were dichotomous (e.g. stunted or not stunted, underweight or not underweight, and thin or not thin), binominal logistic regression was conducted as the multivariable analyses to control potential confounding variables.

\section{Ethical consideration}

The study protocol was submitted to the National Committee for Biomedical Research Ethics, the Malagasy Ministry of Health, for its ethical clearance. Yet, the Committee officially approved the study protocol by exempting it from an ethical clearance process, due to low level invasiveness of the study design (Exemption letter No. 52).

It was found that all the first and second graders at the target 10 primary schools were under 16 years of age, by reviewing the student registries. Therefore, an advanced informed consent to participate in both anthropometric measurements and structured household interviews was obtained in written form from the parents of each sampled schoolchild through teaching staff of the primary schools. In addition, a verbal informed consent to participate in the study was further obtained from the parents or other household members responsible for food preparation upon a household visit, in case they change their minds.

\section{Results}

Of 404 children sampled, five were dropped due to parental refusal or absence at their households despite successful contacts with children at schools. It was found that six of 399 children were under 5 years of age. Thus, those six children were excluded from the analysis. As a result, data collected from 393 children and their household members were analyzed. WHO growth reference does not cover children older than 120.8 months of age in z-score calculation for weight-for-age. Therefore, 11 children categorized into the age group were excluded from estimation of prevalence of underweight [16].

The overall prevalence rates of stunting, underweight and thinness were $34.9 \%$ [95\% CI: 30.4-39.7\%], 36.9\% [95\% CI: $32.2-41.9 \%$ ] and $11.2 \%$ [95\% CI: 8.5-14.7\%], respectively (Table 1 [7, 8, 18-20] and Fig. 1). Of 393 children assessed in this study, 123 (31.2\%) suffered from multiple forms of malnutrition (Fig. 2). Note that 19 children (4.8\%) suffered from all three forms of undernutrition. Prevalence rates of overweight and obesity were $1.0 \%$ [95\% CI: 0.4-2.6\%] and 0\% [95\% CI: $0.0-$ $1.0 \%]$, respectively. Thus, overnutrition among schoolaged children in Antananarivo-Avaradrano district need not be addressed as a major public health issue. For this reason, further analyses were conducted only for stunting, underweight and thinness in search for their possible risk factors.

The background variables were compared between stunted and not stunted children, between underweight and not underweight children, and between thin and not thin children. Of 51 background variables, five produced significant associations $(P<0.05)$ with whether being stunted in bivariate analyses (Tables 2 and 3). Similarly, seven and three background variables produced significant associations $(P<0.05)$ with whether being underweight and with whether being thin, respectively. Moreover, multicollinearity was not confirmed, as only VIF $<10$ was detected among them. Therefore, all these background variables with significant associations were employed as the independent variables for logistic regressions.

The results of logistic regressions are shown in Table 4. In the logistic regression on being stunted, four of five independent variables produced significant adjusted odds ratios (ORs) $(P<0.05)$, i.e. 'Seller, trader or commercial business', 'Sweets', 'Age', and 'Total number of household members'. In the logistic regression on being underweight, four of seven independent variables produced significant adjusted ORs $(P<0.05)$, i.e. 'Seller, trader or commercial business', 'Age,' 'Total number of household members', and 'Household dietary diversity score'. In the logistic regression on being thin, two of three independent variables produced significant ORs $(P<0.05)$, i.e. 'Age' and 'Total number of household members'.

\section{Discussion}

Prevalence of stunting among children aged 5-14 years $(34.9 \%)$ was lower than national prevalence of stunting among children under 5years of age (49.2\%) [8]. Its data as of 2008-2016, during which the children assessed in this study used to be under 5 years of age, were consistently around 50\%, too [18]. Yet, the data specific to Antananarivo-Avaradrano district as of 2008-2016 is not available. We should assume that prevalence of stunting during their under-five childhood was at the same level as the current figure, as catch-up in height-for-age during 24-59 months of age is generally unexpectable $[10,14]$. 


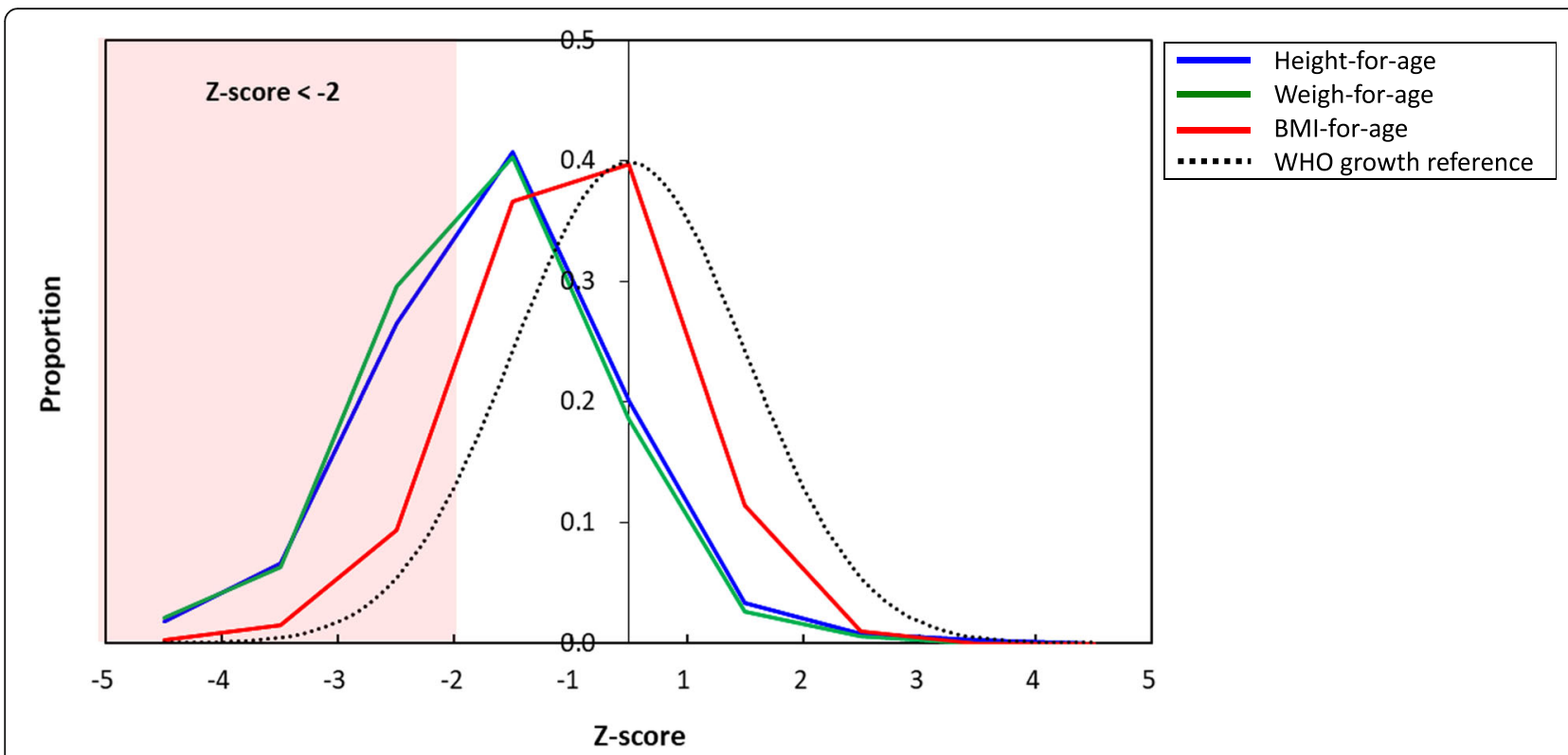

Fig. 1 Frequency distributions of z-scores for height-for-age, weight-for-age and BMl-for-age among schoolchildren 5-14 years of age

Prevalence of underweight among children aged 5-14 years $(36.9 \%)$ was at the same level as national prevalence of underweight among children under 5 years of age (36.0\%). Prevalence of thinness among children $(11.2 \%)$ was lower than those of stunting and underweight. Absence of national and provincial prevalence of thinness makes it difficult to compare this result with others. This is because prevalence of thinness has never been reported from Madagascar. Prevalence of thinness is a malnutrition indicator applicable exclusively for 519 years of age. Therefore, the indicator is rarely assessed. Note that a majority of nutrition surveys target not school-aged children and older age groups but exclusively children under 5 years of age and their mothers. It is not surprising to learn that the relatively new indicator is generally not attractive enough for health ministries to monitor its data, as it has not been employed as an indicator for global agenda such as SDGs [1].

'Age' produced significant ORs $>1 \quad(P<0.05)$ in all three logistic regression models for stunting, underweight and thinness (Table 4). This is consistent with the increase in prevalence of stunting, underweight and thinness according to age (Fig. 3). Earlier studies reported that z-score of height-for-age either remains low or even further drops during the period from two to 14 years of age in low- and middle-income countries. It is only adolescence (i.e. $14-20$ years of age) when catch-up

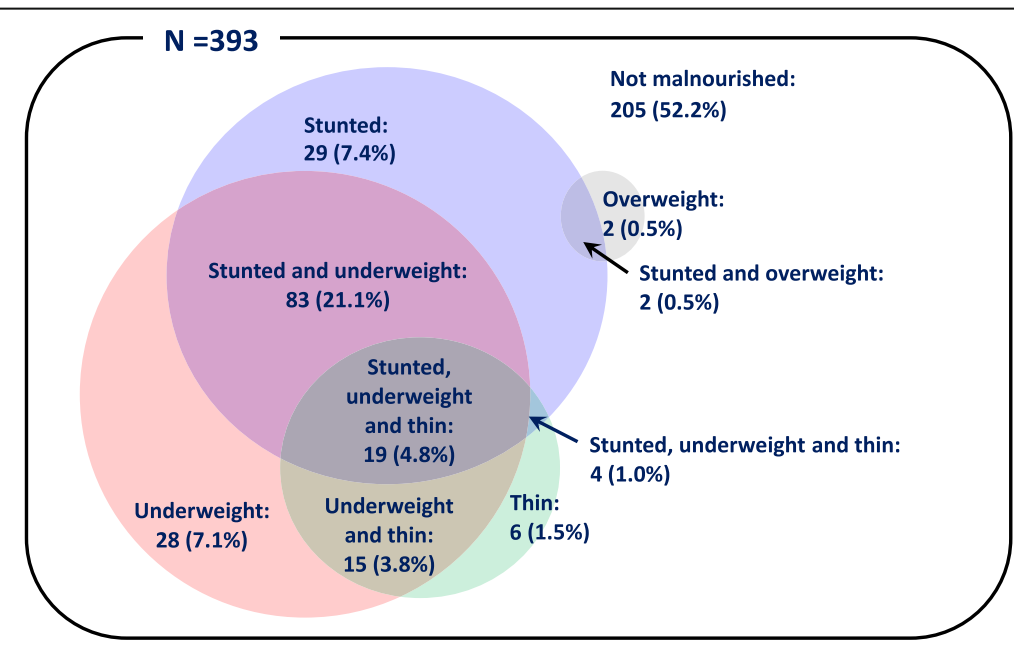

Fig. 2 Multiple forms of malnutrition among schoolchildren 5-14 years of age 
Table 4 Logistic regressions on being malnourished with background variables

\begin{tabular}{|c|c|c|c|}
\hline Logistic regression model & Adjusted OR & $95 \% \mathrm{Cl}$ & $P$-value \\
\hline \multicolumn{4}{|l|}{ Logistic regression on being stunted ${ }^{a}$} \\
\hline$v_{5}$ : Seller, trader or commercial business (dummy variable for 'Major income sources') & 2.173 & $1.120-4.219$ & $0.022^{*}$ \\
\hline $\mathrm{V}_{43}$ : Milk and milk products (dummy variable for 'Food consumption during last $24 h^{\prime}$ ') & 0.671 & $0.309-1.458$ & 0.313 \\
\hline $\mathrm{v}_{45}$ : Sweets (dummy variable for 'Food consumption during last $24 h^{\prime}$ ) & 0.616 & $0.382-0.994$ & $0.047^{*}$ \\
\hline$v_{47}:$ Age $[$ year] & 1.392 & $1.164-1.664$ & $<0.001^{* *}$ \\
\hline$v_{48}:$ Total number of household members [person] & 1.172 & $1.031-1.333$ & $0.015^{*}$ \\
\hline \multicolumn{4}{|l|}{ Logistic regression on being underweight ${ }^{\mathrm{b}}$} \\
\hline$v_{5}$ : Seller, trader or commercial business (dummy variable for 'Major income sources') & 2.654 & $1.339-5.261$ & $0.005^{* *}$ \\
\hline$\vee_{25}$ : Plastic bag (dummy variable for 'Rice storage') & 1.923 & $0.962-3.844$ & 0.064 \\
\hline$v_{39}$ : Meats (dummy variable for 'Food consumption during last $24 h$ ') & 0.711 & $0.410-1.233$ & 0.224 \\
\hline $\mathrm{V}_{43}$ : Milk and milk products (dummy variable for 'Food consumption during last $24 h^{\prime}$ ) & 0.725 & $0.321-1.634$ & 0.437 \\
\hline$v_{47}:$ Age [year] & 1.474 & $1.189-1.827$ & $<0.001^{* *}$ \\
\hline$v_{48}:$ Total number of household members [person] & 1.189 & $1.043-1.356$ & $0.010^{*}$ \\
\hline$\vee_{51}:$ Household dietary diversity score (HDDS) [pt] & 0.832 & $0.701-0.993$ & $0.043^{*}$ \\
\hline \multicolumn{4}{|l|}{ Logistic regression on being thinc } \\
\hline$v_{30}$ : In open-space without cleaning (dummy variable for 'Utensil maintenance') & 0.298 & $0.069-1.284$ & 0.104 \\
\hline$v_{47}:$ Age [year] & 1.348 & $1.092-1.663$ & $0.005^{* *}$ \\
\hline$v_{48}:$ Total number of household members [person] & 1.240 & $1.038-1.481$ & $0.018^{*}$ \\
\hline
\end{tabular}

${ }^{*} P<0.05$

**P $<0.01$

${ }^{\text {a }}$ The dichotomous independent variable (i.e. stunted or not stunted)

${ }^{b}$ The dichotomous independent variable (i.e. underweight or not underweight)

'The dichotomous independent variable (i.e. thin or not thin)

in height-for-age could occur after the initial 2 years of life $[10,14]$. Since there remain $4-5$ years for the current first and second graders to enter that age group, impact of strengthening school feeding on height-for-age might not be observable during their primary education enrollments. Nevertheless, adequate food availability and dietary diversity over a sufficient period time including primary education period should be ensured [10, 14]. Thus, strengthening of school feeding will be an essential investment and its evaluation should be conducted not prematurely in a few years but at an appropriate time, i.e. when and/or after first and second graders complete primary education. To increase the likelihood of post-under-two catch-up in both height-for-age and weight-for-age, childhood gastrointestinal infections [14] (schistosomiasis in particular) [27-29] should be adequately prevented and controlled. Madagascar has the world's fifth highest prevalence of schistosomiasis $(52.1 \%$ as of 2010) [30]. WHO recommends that all school-aged children be treated by mass praziquantel administration once a year in schistosomiasis high endemic countries with prevalence $>50 \%$ [31] such as Madagascar. Though 152 of 399 children (38.1\%) assessed in this study underwent school-based deworming, their prevalence of all three forms of undernutrition was not significantly different from those not having undergone it (Table 2).
Irregular and sporadic implementations of school-based deworming program might have made deworming less effective. Of 10 primary schools visited, eight implemented deworming interventions before. Yet, at three of eight primary schools, more than 3 years have passed since the last deworming implementation.

Also, 'Total number of household members' produced significant ORs $>1 \quad(P<0.05)$ in logistic regression models for stunting, underweight, and thinness (Table 4). This indicates that, the greater number of members a household has, the higher likelihood of being stunted, underweight and thin its schoolchild has. There are two possible reasons for this. First, per-capita food availability at larger households should become smaller. Members of larger households naturally can access and consume a less amount of foods than those of small households, when comparing households at the same income level. In addition, the average number of household members in the lowest quintile income group is the greatest of all quintile income groups, in Madagascar [32]. This synchronously makes it difficult for larger households to ensure per-capita food availability. Second, Malagasy households generally tends to allocate a less amount of foods inadequately meeting age-specific energy requirement to young and older children [33]. Thus, nutritional deficiencies among schoolchildren in this study might be 


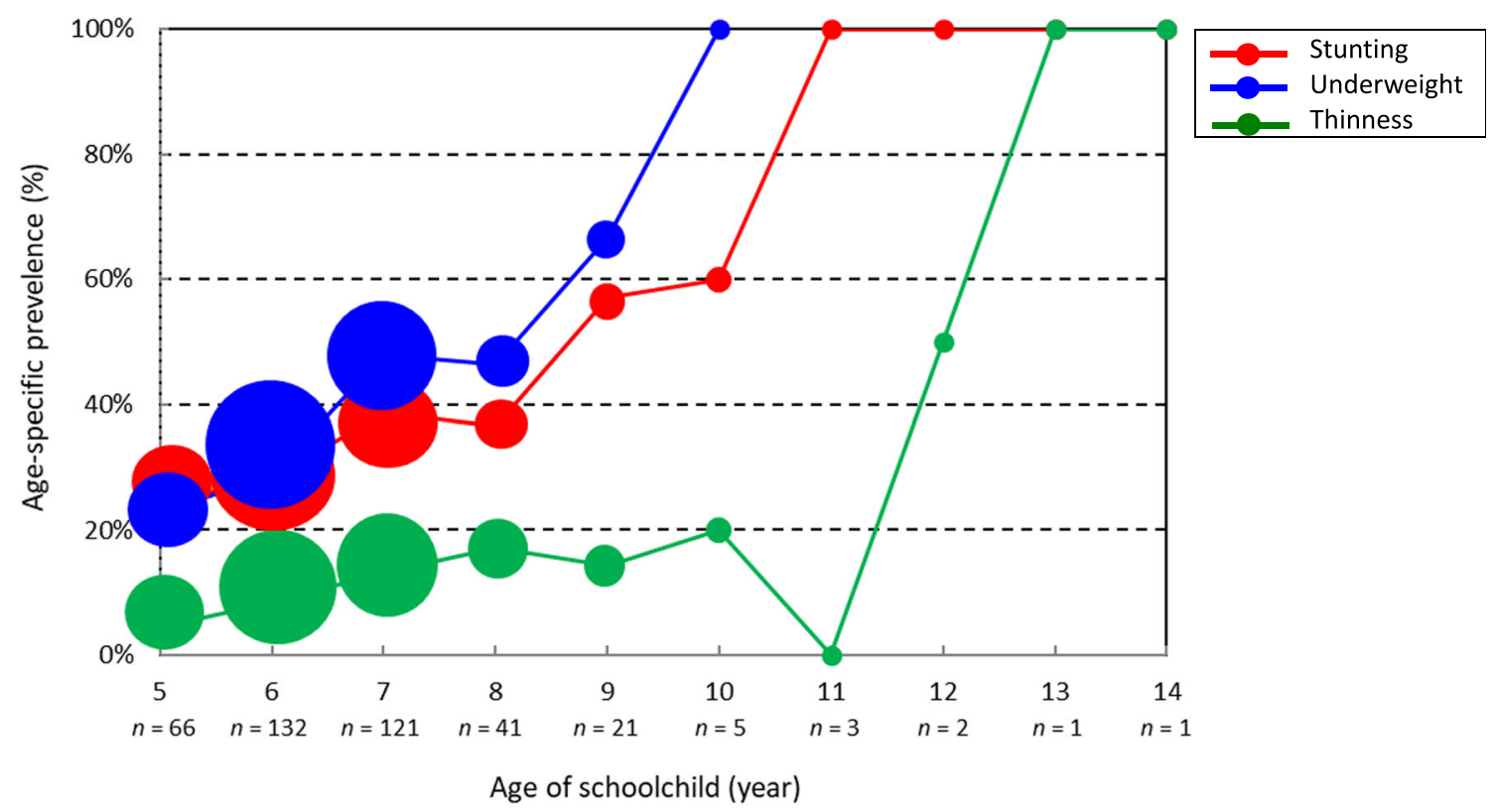

Fig. 3 Age-specific prevalence of stunting, underweight and thinness among schoolchildren 5-14 years of age. [Remarks] Sizes of respective plots are in proportion to total number of children

more severe than among their parents. Having assumed this, school-feeding should play a key role as the critical nutrient source supplementary to children's inadequate diet at household level derived from inappropriate intrahousehold food distribution.

'Household dietary diversity score (HDDS)' produced significant $\mathrm{OR}<1 \quad(P<0.05)$ in the logistic regression model for underweight (Table 4). This indicates that children consuming a fewer number of food groups are likely to be underweight. Inadequate intake of animalbased protein ingredients ('Meats', 'Milk and milk products') were significantly associated with being stunted and underweight $(P<0.05)$ in bivariate analyses (Table $2)$, though these variables did not produce significant OR $(P<0.05)$ (Table 4$)$. This may imply that there might be a need for improvement of dietary diversity through using particularly animal-based protein ingredients for school meals. Those having 'had lunch through school feeding program' on the previous day of anthropometric measurements had significantly greater HDDS than those not having $(P<0.05$ : Mann-Whitney $U$ test $)$. Yet, its mean difference between the two groups was only 1.12 (=7.17-6.05). In fact, 'had lunch through school feeding program' was not associated with being stunted, underweight and thin. These support a need for improvement in school feeding program by increasing the number of food groups in school meals.

This study has limitations in accuracy in schoolchildren's individual dietary data, as parental 24-h diet recalls and school feeding program records were sources of the data. Yet, monotonous dietary pattern in rural
Malagasy households is generally likely to ensure a certain generalizability of 24-h dietary not only over a period of time but also to all household members. Note that parents of 384 children in this study $(97.7 \%)$ stated that dietary pattern of last 4 days remained the same. Moreover, earlier studies found that parents are reliable reporters at least of their children's in-home dietary intake [34].

\section{Conclusions}

Prevalence rates of stunting and underweight among 514 years of age were as high as those among children under 5 years of age. Difference in the definitions between thinness among children 5-14 years of age and wasting among children under 5 years of age makes it difficult to compare their prevalence rates.

'Age' and 'Total number of household members' were identified as the common possible risk factors for stunting, underweight and thinness among schoolchildren aged 5-14 years. Adequate food availability and dietary diversity over a sufficient period time including primary education period should be necessary for increasing likelihood of catch-up expectable during adolescence. Malagasy households have strong preference for rice and cannot afford to buy meats except on the days when they receive income [35]. School feeding program, therefore, should be able to more feasibly procure animalprotein ingredients than households, by leveraging economies of scale in food procurement.

Nevertheless, household feeding practices should improve in parallel, as school feeding can cover only 172 
days (Malagasy standard number of school days) out of 365 days (47\%) at a maximum in a year. Efforts on feeding practices need to be made both at school and household levels up to 14 years of age and beyond. Note that this study is expected to contribute to evidence-based menu planning for the school feeding program, by serving as the first study that reports risk factors and profiles for malnutrition of school-aged children in Madagascar.

\section{Additional file}

Additional file 1: English version of the questionnaire for the household survey. (PDF $446 \mathrm{~kb}$ )

\section{Abbreviations}

HDDS: Household Dietary Diversity Score; JICA: Japan International Cooperation Agency; MDGs: Millennium Development Goals; MoNE: Ministry of National Education; OR: Odds ratio; PNAN III: The third National Nutrition Action Plan; SDGs: Sustainable Development Goals; SUN: Scaling Up Nutrition; WHO: World Health Organization

\section{Acknowledgments}

The authors gratefully thank Nobuhiro Kunieda for his valuable technical supports. The authors also thank Japan International Cooperation Agency (JICA) for their funding support to the study. This work is sincerely dedicated to all the school-aged children, their parents and caregivers, and teaching staff of primary schools in rural areas of Madagascar.

\section{Authors' contributions}

All the authors made substantial intellectual contributions to the study. HA and KA conceptualized and designed the study. RE, HA, KA and RRA collected data. HA and TM analyzed and interpreted the data. HA drafted and finalized the manuscript. KA, AVN, RE, RRA, TM and MH critically commented and revised manuscript. All the authors reviewed and approved the final version of the manuscript.

\section{Funding}

This work was supported by Japan International Cooperation Agency (JICA) The funding body for this study was involved in study conceptualization and designing, data collection and analysis, and manuscript writing, since the corresponding author having taken those responsibilities is affiliated with the funding body.

\section{Availability of data and materials}

The datasets used and/or analyzed during the current study are available from the corresponding author on reasonable request.

\section{Ethics approval and consent to participate}

The study protocol was submitted to the National Committee for Biomedical Research Ethics, the Malagasy Ministry of Health, for its ethical clearance. Yet, the Committee officially approved the study protocol by exempting it from an ethical clearance process, due to low level invasiveness of the study design (Exemption letter No. 52). An advanced informed consent to participate in both anthropometric measurements and structured household interviews was obtained in written form from the parents of each sampled schoolchild.

\section{Consent for publication}

Similarly to consent to participate, an advanced informed consent for publication was obtained in written form from the parents of each sampled schoolchild..

\section{Competing interests}

The authors declare that they have no competing interests.

\section{Author details}

'Human Development Department, Japan International Cooperation Agency (JICA), 3rd floor, Nibancho Center Building, 5-25 Niban-cho, Chiyoda-ku, Tokyo 102-8012, Japan. ${ }^{2}$ Department of Global Health, Milken Institute
School of Public Health, The George Washington University, 950 New Hampshire Ave, NW, 7th floor, Washington, DC 20052, USA. ${ }^{3}$ JICA Participatory and Decentralized School Management Support Project, INFP Mahamasina Porte, 215 Antananarivo, Madagascar. ${ }^{4}$ Ministry of National Education, Bâtiment Ex-UERP, Immeuble FFM, 1ère étage, porte 115 Bis, Complexe Ampefiloha, 101 Antananarivo, Madagascar. ${ }^{5}$ Association des consultants a la recherche au devellopement economique et social (ACREDES), Ministry of National Education, Ambohidrapeto Lot IAB, $21 \mathrm{G}$ Antananarivo, Madagascar. ${ }^{6}$ International Support and Partnership for Health (ISAPH), P. O. Box 35, Mzimba, Malawi.

Received: 11 November 2018 Accepted: 20 May 2019

Published online: 17 June 2019

\section{References}

1. United Nations (UN). Sustainable development goal 2 - End hunger, achieve food security and improved nutrition, and promote sustainable agriculture. New York: UN; 2015. https://sustainabledevelopment.un.org/sdg2 (accessed 19 Oct 2018)

2. United Nations (UN). Millennium development goal 1 - Eradicate extreme poverty and hunger. New York: UN; 1990. http://www.un.org/ millenniumgoals/poverty.shtml (accessed 19 Oct 2018)

3. Scaling up nutrition (SUN). SUN movement monitoring, evaluation, accountability, and learning. Geneva: SUN; 2017

4. Best C, Neufingerl N, van Geel L, van den Briel T, Osendarp S. The nutritional status of school-aged children: why should we care? Food Nutr Bull. 2010; 31(3):400-17.

5. World Bank. Human capital summit 2017 - Committing to Action to Drive Economic Growth. Washington DC: World Bank; 2017. http://www. worldbank.org/en/news/feature/2017/10/20/countries-commit-to-strongaction-on-human-capital-to-drive-economic-growth (accessed 19 Oct 2018)

6. World Health Organization (WHO). The growth chart - a tool for use in infant and child health care. Geneva: WHO; 1986

7. de Onis M, Onyango AW, Borghi E, Siyam A, Nishida C, Siekmann J. Development of a WHO growth reference for school-aged children and adolescents. Bull World Health Organ. 2007:85(9):660-7.

8. International Food Policy Research Institute (IFPRI). Global nutrition report 2016 - from promise to impact, ending malnutrition by 2030. Washington DC: IFPRI; 2016.

9. L'Office National de Nutrition (ONN). Plan National d'Action pour la Nutrtionlll 2017-2021. Antananarivo: ONN; 2017. (in French).

10. Victora CG, de Onis M, Hallal PC, Blössner M, Shrimpton R. Worldwide timing of growth faltering: revisiting implications for interventions. Pediatrics. 2010;125(3):e473-80

11. World Food Programme (WFP). Madagascar-comprehensive food security and vulnerability analysis. Rome: WFP; 2006

12. Institut National des Statistiques. Recensement General de la Population et de l'habitation. Institut National des Statistiques. Antananarivo: Institut National des Statistiques; 1993. (in French)

13. Institut National des Statistiques. Monographie d'Antananarivo, UPDR/ Ministère de l'Agriculture, de l'Elevage et de la Pêche. Antananarivo: Institut National des Statistiques; 2005. (in French)

14. Prentice AM, Ward KA, Goldberg GR, Jarjou LM, Moore SE, Fulford AJ, Prentice A. Critical windows for nutritional interventions against stunting. Am J Clin Nutr. 2013;97(5):911-8.

15. Golden MHN. Is complete catch-up possible for stunted malnourished children? Eur J Clin Nutr. 1994;48(Suppl 1):S58-70.

16. World Health Organization (WHO). AnthroPlus for personal computers manual. Geneva: WHO; 2009. http://www.who.int/growthref/tools/who_ anthroplus manual.pdf?ua=1 (accessed 19 Oct 2018)

17. ACT watch. Household survey Madagascar 2012 Survey Report. Washington DC: ACT watch: 2013

18. World Health Organization (WHO). Computation of centiles and z-scores for height-for-age, weight-for-age and BMI-for-age. Geneva: WHO; 2007.

19. United Nations Children's fund (UNICEF). Madagascar-Statistics. New York: UNICEF; 2018. https://www.unicef.org/infobycountry/madagascar_statistics. html (accessed 19 Oct 2018)

20. World Bank. World Bank open data. Washington DC: World Bank; 2018. https://data.worldbank.org/indicator/SH.STA.OWGH.ZS?locations=MG\&view= chart (accessed 19 Oct 2018)

21. Food and Agriculture Organization of the United Nations (FAO). Guidelines for measuring household and individual dietary diversity. Rome: FAO; 2010. 
22. United Nations Children's Fund (UNICEF). Improving child nutrition: The achievable imperative for global progress. New York: UNICEF; 2013.

23. Aiga H, Matsuoka S, Kuroiwa C, Yamamoto S. Malnutrition among the children in the rural Malawian fish-farming households. Trans R Soc Trop Med Hyg. 2009;103(8):827-33.

24. Negishi H. Vulnerability of maternal and child diet habit - strong impact of school feeing and challenges of agricultural cooperatives. Monthly Rev Agric Forestry Fishery Finance. 1990;43(12):796-805 (in Japanese).

25. R Core Team. R: a language and environment for statistical computing. Vienna: R Foundation for Statistical Computing; 2018. https://www.R-project. org/ (accessed 8 May 2019).

26. IBM. IBM SPSS Statistics and open source: A powerful combination; 2018 https://www.ibm.com/downloads/cas/YB9DVOAR (accessed 8 May 2019).

27. Stoltzfus RJ, Albonico M, Tielsch JM, Chwaya HM, Savioli L. School-based deworming program yields small improvement in growth of Zanzibari school children after one year. J Nutr. 1997;127(11):2187-93.

28. Frigerio S, Macario M, lacovone G, Dussey-Comlavi KJ, Narcisi P, Ndiaye AT, Moramarco S, Alvaro R, Palombi L, Buonomo E. Schistosoma haematobium infection, health and nutritional status in school-age children in a rural setting in northern Senegal. Minerva Pediatr. 2016;68(4):282-7.

29. Kinung'hi SM, Mazigo HD, Dunne DW, Kepha S, Kaatano G, Kishamawe C, Ndokeji S, Angelo T, Nuwaha F. Coinfection of intestinal schistosomiasis and malaria and fassociation with haemoglobin levels and nutritional status in school children in Mara region, Northwestern Tanzania: a cross-sectional exploratory study. BMC Res Notes. 2017;10(1):583.

30. Rollinson D, Knopp S, Levitz S, Stothard JR, Tchuem Tchuenté LA, Garba A Mohammed KA, Schur N, Person B, Colley DG, Utzinger J. Time to set the agenda for schistosomiasis elimination. Acta Trop. 2013;128(2):423-40.

31. World Health Organization (WHO). Prevention and control of schistosomiasis and soil-transmitted helminthiasis: report of a WHO expert committee. WHO tech. Rep. Ser. 912. Geneva: WHO; 2002. p. 1-57.

32. International Monetary Fund (IMF). Republic of Madagascar Economic Development Document-IMF Country Report No. 17/225. Washington DC: IMF; 2017.

33. Berti PR. Intrahousehold distribution of food: a review of the literature and discussion of the implications for food fortification programs. Food Nutr Bull. 2012;33((3 Suppl):S163-9.

34. Klesges RC, Klesges LM, Brown G, Frank GC. Validation of the 24-hour dietary recall in preschool children. J Am Diet Assoc. 1987;87(10):1383-5.

35. Ramaroson RV, Arvisenet G, Valentin D. Studying the nutritional beliefs and food practices of Malagasy school children parents. A contribution to the understanding of malnutrition in Madagascar. Appetite. 2014;81:67-75.

\section{Publisher's Note}

Springer Nature remains neutral with regard to jurisdictional claims in published maps and institutional affiliations.

Ready to submit your research? Choose BMC and benefit from:

- fast, convenient online submission

- thorough peer review by experienced researchers in your field

- rapid publication on acceptance

- support for research data, including large and complex data types

- gold Open Access which fosters wider collaboration and increased citations

- maximum visibility for your research: over $100 \mathrm{M}$ website views per year

At $\mathrm{BMC}$, research is always in progress.

Learn more biomedcentral.com/submissions 